\title{
Time evolution of aqueous foams: drainage and coarsening
}

\author{
Arnaud Saint-Jalmes and Dominique Langevin \\ Laboratoire de Physique des Solides, Université Paris-Sud, 91405 Orsay, France
}

Received 1 May 2002, in final form 21 June 2002

Published 27 September 2002

Online at stacks.iop.org/JPhysCM/14/9397

\begin{abstract}
We report new results on drainage and coarsening of aqueous foams. We show that these two effects can strongly interfere, enhancing the drainage velocity. Without coarsening, we have performed free-drainage experiments, in which local drainage rates are measured by electrical conductivity and by light scattering techniques. We have investigated the roles of the bubble size, of the surface and bulk rheology and of the liquid fraction. The results show that changing these foam parameters can induce transitions between different drainage regimes. The results are analysed in terms of two dimensionless numbers describing the balance between surface and bulk dissipation.
\end{abstract}

\section{Introduction}

Aqueous foams are dispersions of gas in liquid, stabilized by surfactant adsorbed at the airliquid interfaces [1]. If we look around us, aqueous or solid foams are ubiquitous in everyday life (cosmetics, food, detergents, packaging etc). Beside the evident importance of their applications, aqueous foams are also interesting to study as model systems of a new class of materials called soft glassy materials, which include emulsions, slurries, pastes or granular systems.

A main property of aqueous foams is that they are out-of-equilibrium systems. They evolve in time by gravitational drainage, coarsening and film rupture [1]. Despite the fact that the observation of this time evolution is quite easy, many questions remain unsolved on the mechanisms of these instabilities, and still today no clear picture emerges about what sets a foam lifetime. However, during recent years, this field has been very active, especially regarding drainage, with advances both in theory and in experiments [2-25].

There are two experimental methods for the study of drainage. In the 'free-drainage' situation, a foam of initially uniform and constant wetness is allowed to drain: the foam dries first at the top, and a dry front propagates down through the foam, while the liquid emerges and accumulates at the bottom. Alternatively, in the 'forced-drainage' method, the surfactant solution is poured onto the top of an already dried foam, and a wet front propagates down through the foam. 
In both cases, in order to understand the properties of these macroscopic behaviours, one needs to come to a more detailed description of the foam, at the scale of the bubbles. For the drainage problem, one usually models the foam as a network of interconnected channels, called the plateau borders (PBs), with the surfactant solution flowing along this network. These PBs are connected in fours at vertices, or nodes. The drainage problem recalls the problem of flow in porous media, but with the specificity that the PB cross section varies with the liquid content: bubbles are deformable objects, and can be more or less packed depending on the foam wetness. In a single PB, the gravitational force is balanced by capillarity (which tends to bring the liquid from wet to dry zones), and by different viscous dissipation effects. These dissipative effects and the balance between them are strongly connected to the boundary conditions at the PB surfaces (more or less mobile). One of the major issues today in this field is to understand what sets the boundary conditions (thus the flow regime), and how this varies with the foam properties (bubble size, foam wetness, surfactant used and solution used). Also, one has to understand the type of dissipation associated with the different boundary conditions and macroscopic behaviours. The classical approach to the problem, being based on models at the PB scale in order to predict the drainage behaviour at a much larger macroscopic scale, relies on some basic assumptions which have to be tested, and the limits of such an approach need to be investigated (especially for the case of very wet foams, where the PBs and nodes are no longer well defined).

Another important issue is to elucidate the effect of the gas, which affects the coarsening process. Foams coarsen because of pressure differences between bubbles of different sizes. This results in an increase of the mean bubble size. Coarsening and drainage may interfere, depending on the timescales of these two effects. Recent results have shown that coarsening can enhance drainage, but some questions remain unsolved, especially about the dependence of the coarsening rate on the liquid fraction, and about the mechanisms of drainage enhancement.

Despite its simplicity, free drainage is more difficult to analyse than forced drainage, and such studies have not yet been able to produce comprehensive and reproducible sets of results. It is important to note that the coupling with coarsening is not the only effect making free-drainage experiments more difficult to perform and to interpret. The foam uniformity, measurement methods and the treatment of the boundary conditions at the foam top and bottom are also problems which need to be carefully handled in order to finally make the link between free and forced drainage.

Clarifying all these issues, and understanding the effects of the foam basic constituents and basic parameters (such as the bubble size, or the wetness) is indeed the first necessary step for further studies of more complex foaming solutions, as well as for a good control and understanding of foam rheology measurements.

In the first section of this paper, we present the different types of boundary condition at the PB surfaces, and the related drainage regimes, discussed in terms of the balance between surface and bulk viscous dissipation effects. Then we present our experimental approach and the results of controlled free-drainage experiments showing how gas, bubble size, bulk viscosity, surface rheology and wetness of the foam affect drainage, and induce drainage regime transitions. We have focused our studies on small bubbles (with diameter $D<1 \mathrm{~mm}$ ), a relatively uncovered range of size in these studies. In the discussion section, we compile our results with previous ones on larger bubble sizes, and try to answer some of the outstanding issues presented above.

\section{Boundary conditions and drainage regimes}

A crucial point in the description of the flow in PBs is the treatment of the boundary conditions at their surfaces. These surfaces can be immobile and rigid so that the flow in the PB is 
Poiseuille-like. Alternatively, the surfaces can also be more fluid and mobile, flowing with the bulk liquid, and providing a plug flow in the PB. This surface mobility is indeed an important property because two different macroscopic regimes of drainage are set by these two mobility limits. Experimentally, these two drainage regimes have been seen for different surfactant systems $[2,13,26]$. It has been shown that a transition from one regime to the other is possible by changing the surface viscoelasticity via mixtures of surfactants [20, 22]. The recent direct observation (via confocal microscopy) of the flow in a single PB has also shown that, depending on the surface viscoelasticity, rigid or mobile surfaces could be found [27]. Changing the surface viscoelasticity can therefore induce the transition from one regime to the other; it is important to understand whether other foam parameters can also control these regimes and the surface mobility. In fact, this question is related to the understanding of the dissipation mechanisms in the foam. In the case of immobile solid surfaces, the main dissipative effect is a bulk one, related to the bulk viscosity of the solution. However, the PB surfaces are viscoelastic, and one must always consider the coupling between the flow within the PB and the flow in its surface (eventually providing surface dissipation). A first type of surface dissipation is related to the transverse shear of the surfaces [28, 29]. Physically, the shear comes from the triangular-like shape of the PB, inducing no speed in its corners, and thus producing a shear within the surface.

The dimensionless number $M_{l}$

$$
M_{l}=\mu r / \mu_{s}
$$

describes the balance between this surface dissipation effect and the bulk viscous dissipation: $\mu$ is the bulk viscosity, $\mu_{s}$ the surface shear viscosity and $r$ the radius of curvature of the PB (proportional to the bubble diameter $D$ and to the liquid fraction $\varepsilon, r \sim D \varepsilon^{1 / 2}$ ). Note that $M_{l}$ is the inverse of the Boussinesq number. The value of $M_{l}$ sets the boundary conditions and the flow type: when $M_{l}<1$, Poiseuille flows are predicted, while for $M_{l}>1$ plug flows are predicted.

Durand et al [21] recently proposed another surface contribution, related to longitudinal effects, and to the creation of surface tension gradients. The balance between bulk and surface effects is then described by $M_{d}$ in the case of an insoluble surfactant:

$$
M_{d}=\left(\mu D_{s}\right) /(E r)
$$

where $D_{s}$ is a surface diffusion coefficient, and $E$ is the Gibbs elasticity of the interface. For a soluble surfactant, $D_{s}$ has to be replaced by an effective diffusion coefficient $D_{\text {eff }}$ [21]. Here also, plug flows are predicted when $M_{d}>1$.

One can see that if the surfaces are less elastic or viscous both $M_{l}$ and $M_{d}$ increase, providing transitions from Poiseuille flow to plug flow. Following these ideas, drainage transitions should also be induced by other parameters (such as $\mu$ or $r$ ), without changing surface properties. However, note that the effects of the bubble size (via $r$ ) are opposite in $M_{l}$ and $M_{d}$.

Beside the surface effects, Koehler et al [13] also proposed to take into account the bulk dissipation in the nodes, which could be the dominant effect for the case of mobile surfaces: merging and bending of flows in the nodes can induce a bulk viscous dissipation, which no longer exists in the PBs. In this case, however, it is impossible to describe the transition of the drainage regime in terms of a simple parameter.

For immobile surfaces, the drainage equation was first derived by Weaire and coworkers [2, 5], and independently by Gol'dfarb et al [26]. The associated flow regime can be recognized in many ways whether it is in the free- or in the forced-drainage experimental mode. In forced-drainage experiments, the liquid front speed $v$ is related to the constant liquid 
flow rate $Q$ via

$$
v \sim Q^{\alpha} \quad \text { with } \alpha=1 / 2 .
$$

In free drainage, we have focused on the time variation of the liquid fraction $\varepsilon$ within a foam at different heights, which follows power laws: $\varepsilon(z)=t^{\beta}$. The exponent $\beta$ depends on the parameter $\chi=z / z_{0}\left(t_{0} / t\right)^{1 / 2}$ [14] ( $z=0$ at the foam top). Here, $t_{0}$ and $z_{0}$ are the typical time and length scales defined by $t_{0}=C \gamma \mu / 2 K_{x}(\rho g)^{2} L^{3}$ and $z_{0}=C \gamma / 2 \rho g L$. $C$ is a constant, $\gamma$ is the surface tension, $\rho$ is the density, $L$ is the PB length $(L \sim D / 3)$ and $K_{x}$ is a dimensionless parameter depending on the drainage regime: $K_{1}$ for rigid surfaces, and $K_{1 / 2}$ for mobile ones [14]. Therefore, for this regime, one finds

$$
\varepsilon \sim t^{-2 / 3} \quad \text { for } \chi<1, \quad \varepsilon \sim t^{-1} \quad \text { for } \chi>1 .
$$

For the other limit (mobile surfaces), it turns out that the drainage equation is identical if one considers that bulk effects in the nodes or longitudinal surface effects in the PBs dominate $[13,14,21]$. This equation provides for forced drainage

$$
v \sim Q^{\alpha} \quad \text { with } \alpha=1 / 3
$$

and for free drainage

$$
\varepsilon \sim t^{-1} \quad \text { for } \chi<1, \quad \varepsilon \sim t^{-2} \quad \text { for } \chi>1 .
$$

For the case of the surface shear effect alone, no analytical calculation is available. However, it seems, in the limit of mobile surfaces, that the drainage regime is different from that found for the two other effects in this same limit (equations (5) and (6)). In fact, numerical simulations show that one could take this effect into account by introducing an acceleration ratio, which is a function of $M_{l}$, and which simply re-scales the coefficient $K_{1}$ [28,30]. In this case, this should result in a regime close to that of rigid surfaces, with a slight increase of $\alpha$ (up to 0.6), and slight decreases of the $\beta$ coefficients. Note that this issue is not completely clarified.

By varying the foam properties (bubble size, solution viscosity and surface rheology), and by determining the conditions to obtain one regime or the other, we can expect to understand what controls the surface mobility, and which dissipation mechanisms are acting.

\section{Experiments: samples, methods and setup}

In our experiments, foams are made by two different techniques. The first one is a turbulent mixing method [17] which provides bubbles with an average size $D(t=0)=180 \mu \mathrm{m}$. The initial liquid fraction $\varepsilon_{0}$ can be controlled and changed from 0.02 to 0.5 . This fast production device ( 11 in $7-8 \mathrm{~s}$ ) is crucial for creating large volumes of initially uniform and homogeneous foams. The uniformity of the foam (meaning an initial liquid fraction constant at any height in the foam) is an important requirement, since initial liquid gradients change the drainage dynamics [17]. In the second method (bubbling method), gas is gently blown through a glass frit into the liquid. This slowly creates the foam, so the foam drains during the production, and the liquid fraction is not uniformly distributed along $z$. To obtain uniform foams, for the freedrainage experiments, we re-wet the foams from the top, at different rates $Q$ (providing different $\left.\varepsilon_{0}\right)$. Here, the bubbles have an average diameter $D(t=0)=800 \mu \mathrm{m}$. The polydispersity is similar in both cases, and remains reasonably small, with no extremely small or large bubbles [17].

We have used two gases: $\mathrm{N}_{2}$ and $\mathrm{C}_{2} \mathrm{~F}_{6}$. The fluorinated gas is highly insoluble in water, and thus strongly reduces coarsening. The surfactant used is sodium dodecyl sulfate (SDS) with or without dodecanol (DOH) (at a weight ratio SDS/DOH $\sim 250$ ). With dodecanol, the 


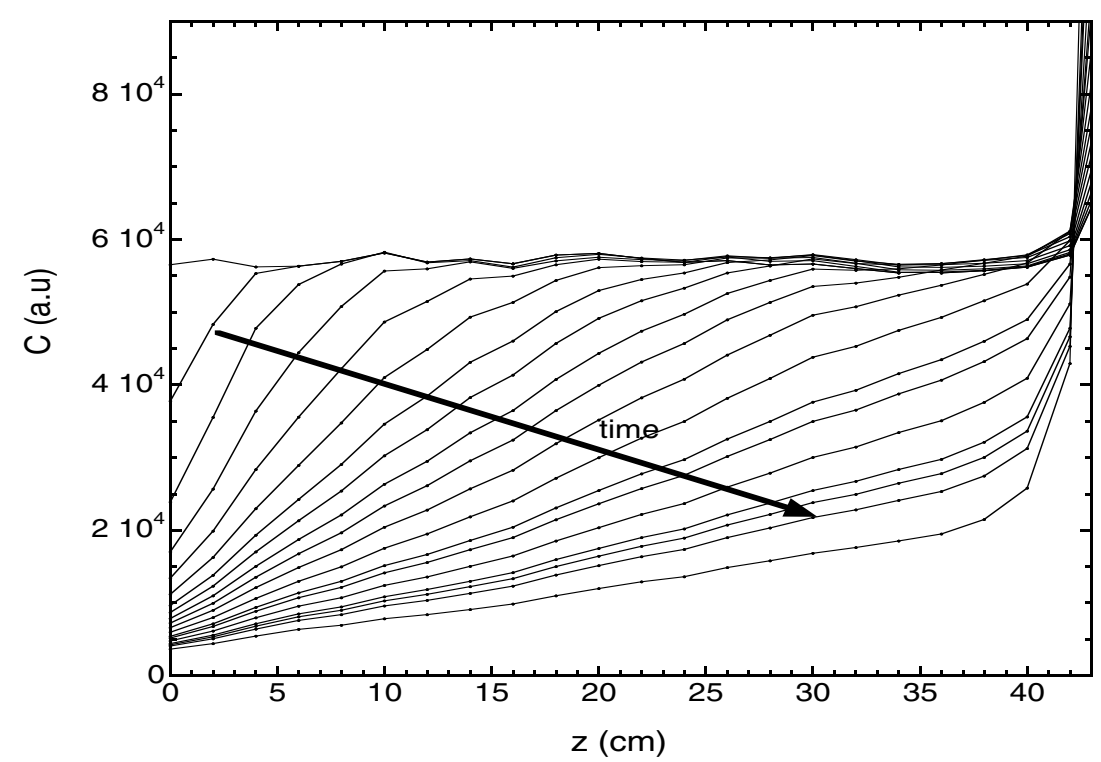

Figure 1. Liquid fraction (proportional to the electrical conductivity) versus height, at different times in the foam. The time between each measurement is $\Delta t=3 \mathrm{~min} . z=0$ at the foam top, $D(t=0)=180 \mu \mathrm{m}$

mixed monolayer at the surface is more elastic and viscous [31]. In some cases, we also have increased the bulk viscosity by adding glycerol to the surfactant solution.

The easiest measurement in free-drainage experiments is the height $L$ of liquid drained out of the foam. This provides the drainage curves $L(t)$, which can be normalized by the final height obtained after complete drainage $L_{f}$. Together with the drainage curves, we follow the drainage dynamics by two complementary techniques. First, we use electrical conductimetry, via a set of electrodes installed along the foam container. The setup is similar to that described in [20,22]. Locally, the liquid fraction $\varepsilon$ is simply proportional to the electrical conductivity $C$. We have measured that this linearity remains valid up to surprisingly high liquid fraction, $\varepsilon \sim 0.25$. Note that not all the questions on the electrical conductivity of foams are resolved today, especially when one wants to obtain an absolute value of the liquid fraction from a single measurement. Here, we use it only for relative variations, and this setup provides the liquid fraction profile in the foam at any time (figure 1). In this graph, one can see that the foam first dries at the top, then deeper in the foam. Note also the creation of a very wet zone near the foam/liquid interface, due to capillarity (the foam locally reaches a high liquid fraction $\sim 0.35$ ). At long times, not all the liquid drains out of the foam, and some liquid remains within an equilibrium vertical profile set by the balance of capillary and gravity forces.

We also use a light scattering method, similar in its concept to that used in [23]. The foam is uniformly illuminated on one side of a flat tall column (height $1 \mathrm{~m}$, thickness $3 \mathrm{~cm}$ and width $25 \mathrm{~cm}$ ) and a CCD camera collects the transmitted light on the other side. For a large enough foam thickness, the light is multiply scattered by the foam, and the transport of photons becomes a diffusive phenomenon. The diffuse transmitted intensity $I_{t}$ is then proportional to $l^{*} / L$, where $l^{*}$ is the mean free path of light in the foam [32]. Using the relationship, $l^{*} / D \sim \varepsilon^{-1 / 2}$ (valid for a large range of bubble size and liquid fraction) [33], one can infer $\varepsilon(t)$ from $I_{t}$, if $D$ is a constant. However, if $D$ is increasing with time by coarsening or coalescence, the transmitted intensity increases both because $D$ increases and $\varepsilon$ decreases. 


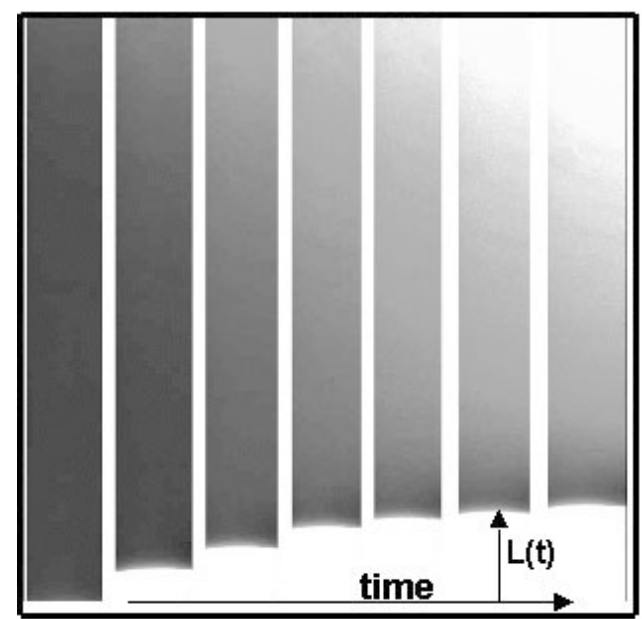

Figure 2. Images in transmission of a foam at different ages: the wetter the foam, the darker the image.

Thus, coupling the two methods on the same sample is an original way to obtain both $\varepsilon(t)$ and $D(t)$, at any position in the foam. Figure 2 shows images of the foam at different ages: in this multiple-scattering regime, the wetter the foam, the darker the picture. The same drainage features as described in figure 1 are recovered here. For all the experiments shown in this paper, the initial height of the foam is constant and is $H \sim 400 \mathrm{~mm}$.

\section{Results}

\subsection{Gas effects and drainage-coarsening coupling}

In order to perform a well controlled free-drainage experiment, we have first investigated the effect of coarsening on the drainage dynamics. It is known that coarsening enhances the drainage $[15,18,23]$ : basically, the bubbles becomes larger, making the PBs wider, thus increasing the drainage speed. However, some questions remain unsolved both on foam coarsening and on the drainage/coarsening coupling.

In dry foams (compacted bubbles), at constant liquid fraction, it has been shown that the bubble size growth law is $R(t) \sim t^{1 / 2}$ ( $R$ is the bubble radius) [34]. This means that the growth rate is simply a function of $\varepsilon$, with a coefficient $G$ depending only on the gas properties:

$$
R(t) \sim t^{1 / 2} \quad \text { and } \quad R \partial_{t} R=G F(\varepsilon) .
$$

This relation between $R(t)$ and $\varepsilon$ (via $F(\varepsilon)$ ) is the key point of the coupling, and the exact form of this function is an important issue today [15, 23]. Also, it is important to know whether the coarsening process depends only on the foam wetness and the gas properties, or whether the adsorbed surfactants at the bubbles interfaces play a role. It seems that there is no surfactant effect when the surfactants are small, but this remains to be tested for mixed and complex polymer/surfactant layers or stacked protein layers. For very wet foams (separated bubbles) the exponent of the growth law is one-third: another important issue is to understand how and for which liquid fraction this exponent shifts to one-half.

Regarding the drainage/coarsening coupling, one can extract from equation (7) a typical coarsening time:

$$
\tau_{c}=R_{0}^{2} / 2 G F(\varepsilon)
$$



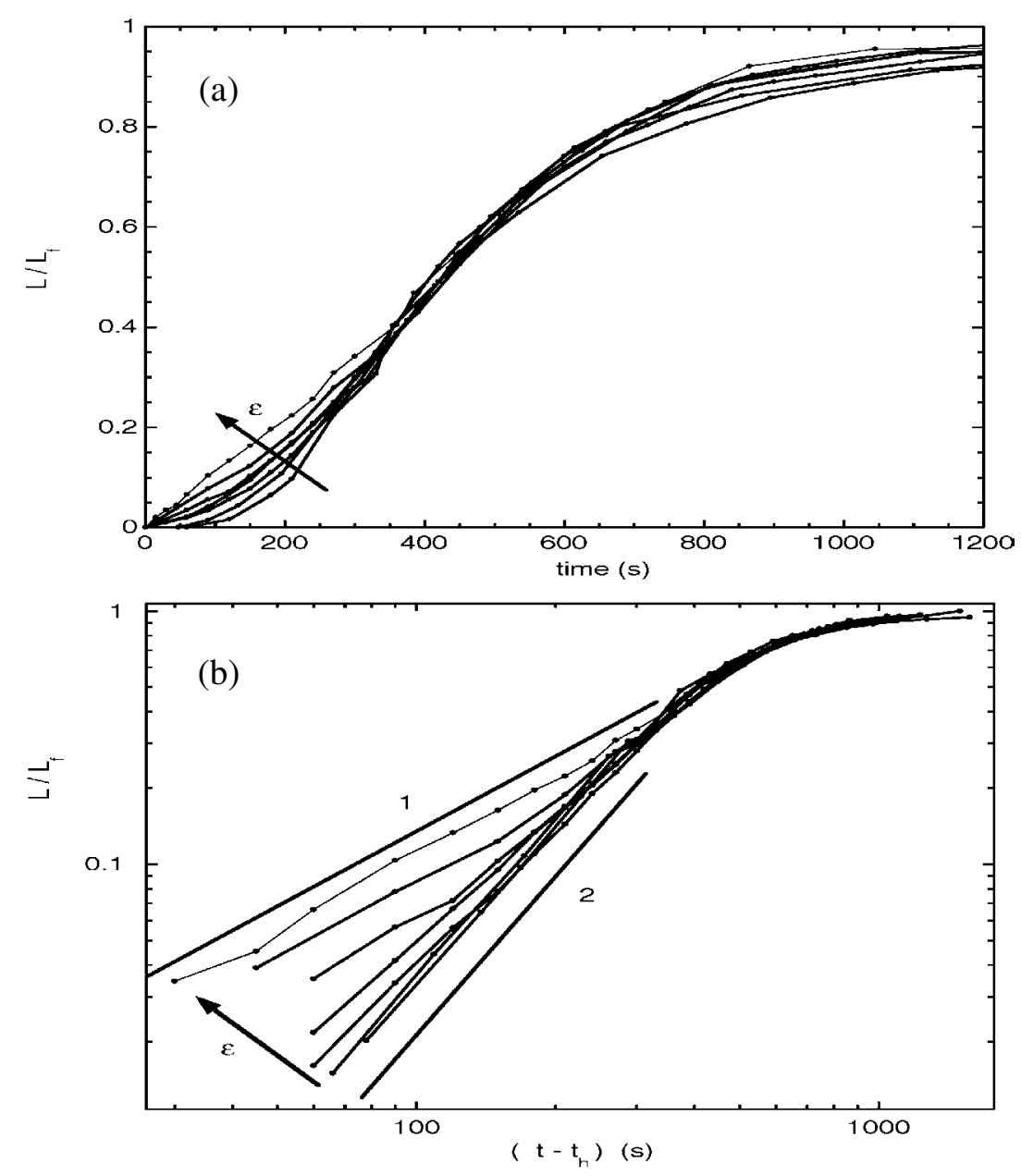

Figure 3. Drainage curves for coarsening foams $\left(\mathrm{N}_{2}\right)$ at different $\varepsilon_{0}$, ranging from 0.04 to 0.3 . The arrows indicate the increase of $\varepsilon_{0} . D(t=0)=180 \mu \mathrm{m}$. (a) $L / L_{f}$ versus time; (b) $L / L_{f}$ versus $t-t_{h}, t_{h}$ being the holdup time.

Comparing $t_{c}$ with the typical drainage times $t_{d}$ (one can use the time when half of the liquid has drained out of the foam) is one way to quantify the importance of coarsening. When $t_{c} \ll t_{d}$ there will be a strong drainage/coarsening coupling, and when $t_{c} \gg t_{d}$ the foam will drain without coarsening. Note that $t_{d} \sim H / R_{0}^{2}$, so $t_{c} / t_{d}$ scales as $R_{0}^{4} / H$, meaning that, beside the gas property $(G)$, the bubble size is also extremely important, as well as the experimental conditions.

From direct observations, our foams made of very small bubbles (size $D=180 \mu \mathrm{m}$ ), and of $\mathrm{N}_{2}$ gas, undergo strong coarsening: the bubbles grow by a factor of five to ten during the drainage time. Figure 3(a) shows the normalized drainage curves for these foams, with $\varepsilon_{0}$ ranging from 0.04 to 0.30 . It first appears that the drainage dynamics hardly depend on the foam wetness. The coarsening is more efficient for the initially drier foams, so it increases their drainage speeds more than for the wettest foams, resulting in less difference between dry and wet foams. Previous studies have shown that the wetness dependence completely disappears for tall enough samples [17]. 


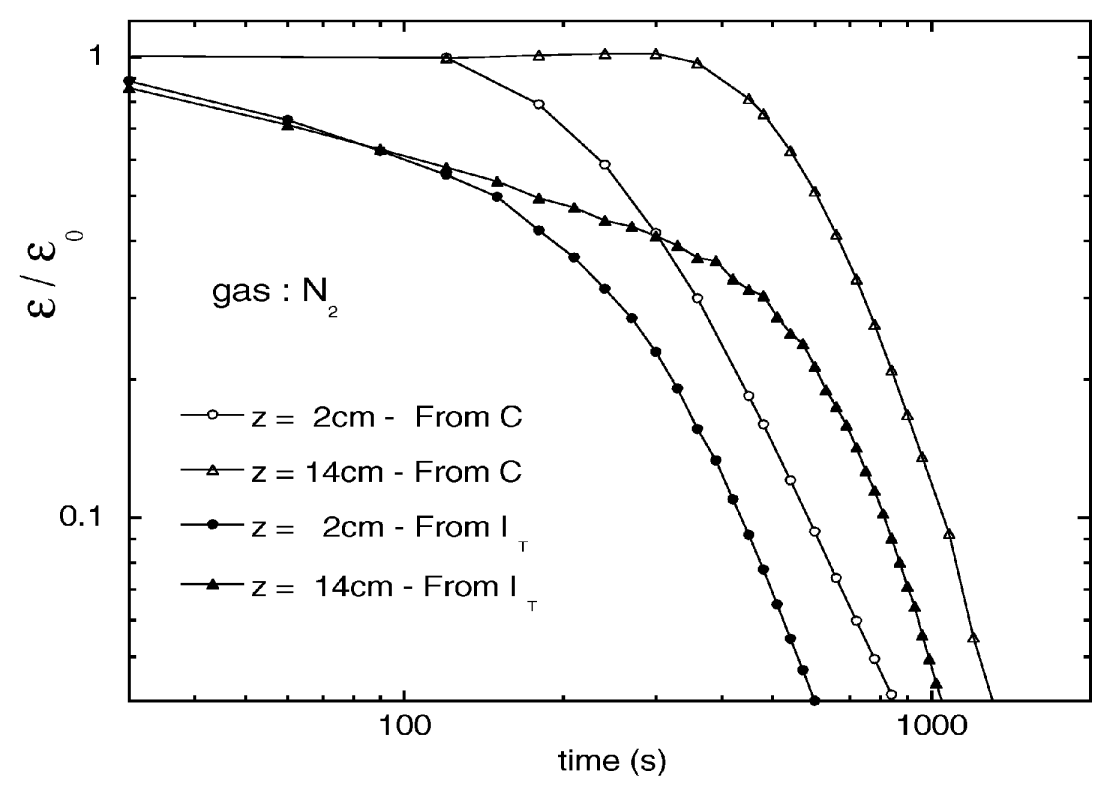

Figure 4. Measurement at two different heights $z$. $D(t=0)=180 \mu \mathrm{m} . \quad \varepsilon_{0}=0.06$. Light scattering data $\left(I_{t}\right)$ treated as if $D=$ const., and electrical conductimetry data $(C)$.

Nevertheless, one can see that at short times there are still differences from $\varepsilon_{0}$ (figure 3(b)). Note that in this figure $L(t)$ is plotted as a function of $t-t_{h}$, where $t_{h}$ is the holdup time. This is the time needed to build the capillary profile at the foam/liquid interface; before $\varepsilon \sim 0.35$ at the interface no liquid can drain out of the foam. $t_{h}$ is thus a decreasing function of $\varepsilon_{0}$, and here is non-zero only for the driest foams, for which $t_{h} \sim 100 \mathrm{~s}$. For the driest foams, a quadratic increase of $L(t)$ is observed, in agreement with predictions for strong coarsening [15]. However, such a behaviour is very difficult to observe: we find that $t_{c} / t_{d}$ must be $\sim 0.15$ to start to see a quadratic increase (when one takes the effective drainage time for $t_{d}$ ). Indeed, the quadratic behaviour transforms into a linear one as $\varepsilon_{0}$ increases, as predicted for small or no coarsening. However, all these foams are still significantly coarsening, regarding the absolute value of their drainage times.

So, it turns out that the observation of features due to coarsening in the shape of the $L(t)$ curves (so, in the drainage rates) are only found when the foam is strongly coarsening, whereas significant decreases of the drainage time are already easily found with small coarsening. This raises some questions on the actual mechanisms of the drainage/coarsening coupling: beside the simple picture related to the bubble growth (equation (7)), other effects could increase the drainage speed [23].

We show in figure 4 results on the local drainage of these $\mathrm{N}_{2}$ foams, obtained with our setup coupling light scattering and electrical conductimetry. We treat the light scattering data as if the bubble size were constant, and obtain what should be the liquid fraction curve. One sees that the two sets of data separate very soon: this is the signature of the bubble growth. This experimental approach appears to be very interesting for answering the previous questions on both foam coarsening and drainage/coarsening coupling. One can have access to the bubble growth behaviour at constant liquid fraction (first decay of the light scattering curve, when the conductivity is still constant), and to the growth behaviour during variation of $\varepsilon$. Checking the consistency of these two regimes should shed light on the coupling mechanism. Moreover, one can also expect to study the stability of thin films: the bubble growth can also be due to film 


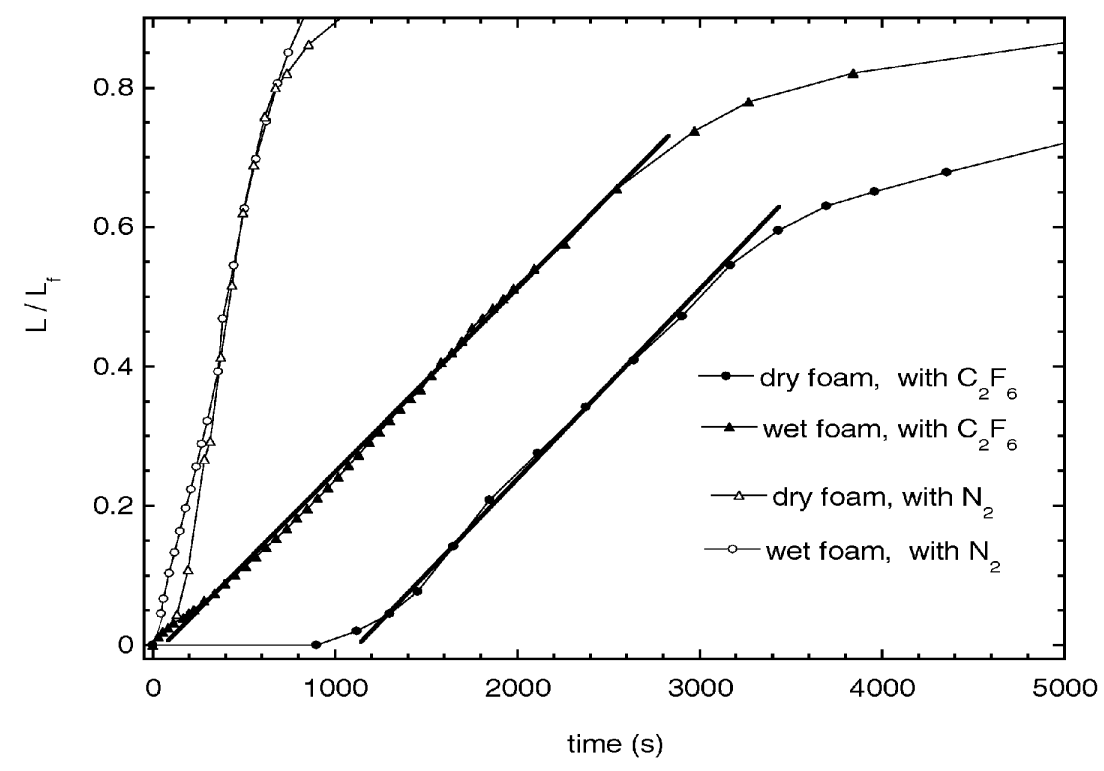

Figure 5. Normalized drainage curves. Comparison between the two gases. $D(t=0)=180 \mu \mathrm{m}$.

rupture (bubble coalescence); without coarsening and at constant liquid fraction, this effect is the only one acting on the light scattering data.

When $\mathrm{C}_{2} \mathrm{~F}_{6}$ is used, there is almost no more coarsening for our bubbles of initial size $D=180 \mu \mathrm{m}$. More precisely, we observe small coarsening, mainly just after foam formation where the bubbles are the smallest: after $30 \mathrm{~min}$, visual observations and light scattering show that the mean bubble size has increased by only $20-25 \%$. At longer times, the bubbles continue to grow, but only slightly and more and more slowly.

Figure 5 shows that with this gas, the drainage times are indeed much longer, and that differences appears with $\varepsilon_{0}$ : the drier the foam, the slower the drainage. Note also that the holdup times become very long for the driest foams, and that linear behaviours are found in $L(t)$.

Local measurements by light scattering and conductimetry show that the two curves are close for the $\mathrm{C}_{2} \mathrm{~F}_{6}$ foams, meaning no significant changes in the bubble size (figure 6 ). One can see that the drainage rates are smaller than for the $\mathrm{N}_{2}$ foams, and that power law regimes are found. Experiments where intermediate coarsening occurs (between the $\mathrm{C}_{2} \mathrm{~F}_{6}$ and $\mathrm{N}_{2}$ foams) confirm our previous results: it is much easier to change the drainage time (making it faster) than to change the drainage rate (in terms of the exponent of the power law regime).

These experiments show that the gas used for the foam is indeed an important and efficient parameter for changing the absolute drainage time. However, the efficiency of the drainage/coarsening coupling depends also strongly on the bubble size, and is really significant for small enough bubbles. Our results also show that coarsening affects the drainage rates only in the very-strong-coarsening regime. We are therefore confident that, for our foams made of $\mathrm{C}_{2} \mathrm{~F}_{6}$ gas, the following free-drainage studies should not be affected by coarsening.

\subsection{Bubble size $D_{1}=800 \mu \mathrm{m}$}

4.2.1. Surface rheology. Figure 7(a) shows the drainage dynamics for SDS/DOH foams $\left(\varepsilon_{0}=0.07\right)$ with bubble size $D_{1}=800 \mu \mathrm{m}$. At long enough times, power law behaviours are observed with exponents $\beta$ increasing from $-2 / 3$ to -1 as $z$ increases. This change of $\beta$ 


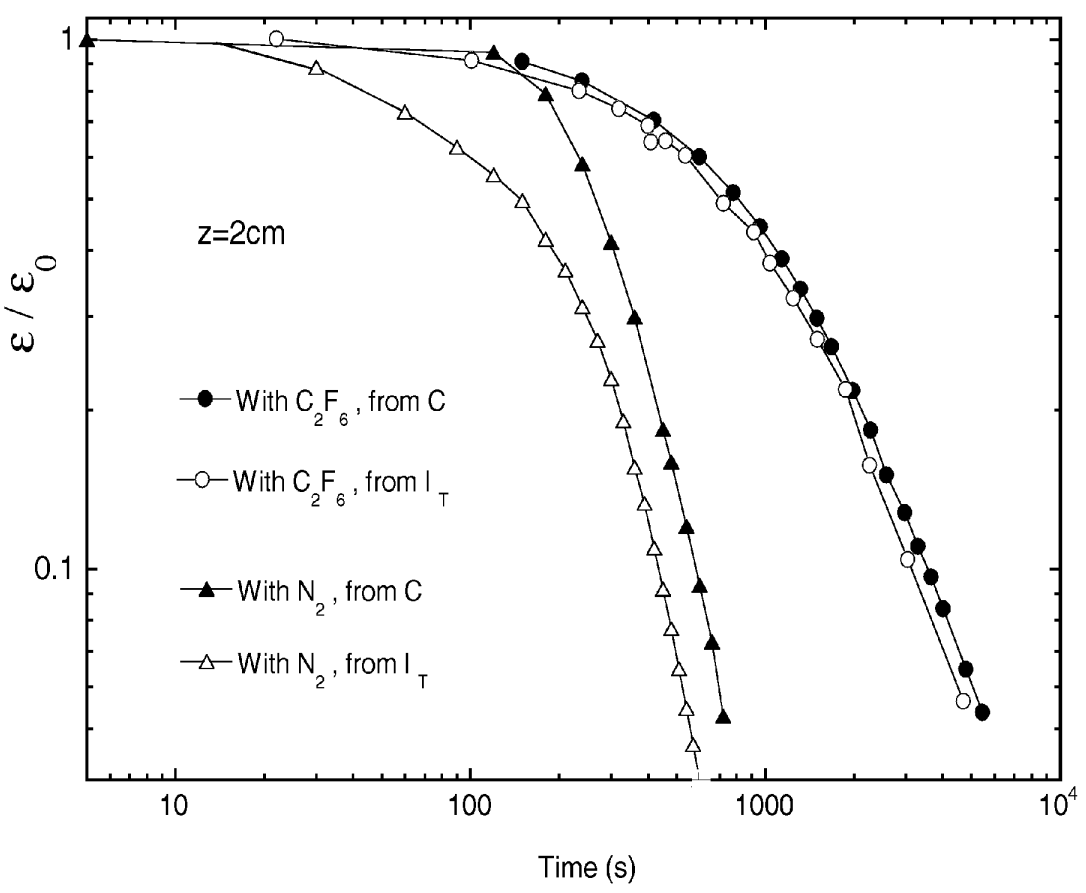

Figure 6. Local measurement. Comparison between the two gases. Circles with $\mathrm{C}_{2} \mathrm{~F}_{6}$; triangles with $\mathrm{N}_{2} ; D(t=0)=180 \mu \mathrm{m} . \varepsilon_{0}=0.08$.

is due to the increase of $\chi$ with $z$ : one can calculate that $\chi>1$ when $z$ is larger than a few centimetres, in agreement with what we found experimentally. Note that $\chi$ changes more with $z$ than with $t$. The exponents are therefore compatible with immobile surfaces, as expected for these rather rigid surfaces (equation (4)). Also in agreement with the immobile surfaces, one can see that near the top the scaling regime starts when $\varepsilon / \varepsilon_{0}<1 / 2$.

The same experiments with pure SDS solutions (less elastic and viscous surfaces) provide similar results, but with exponents ranging from -0.8 to -1.3 . Thus, it appears that the mobile surface regime (see equation (6)) cannot be fully reached with these bubbles (SDS was recrystallized several times to ensure that no residual DOH was present). Forced-drainage experiments with the same bubbles also show that the exponent $\alpha$ never goes below 0.40 for pure SDS.

4.2.2. Bulk viscosity. We have tested the effect on the drainage regime of the bulk viscosity $\mu$ for these foams of bubble size $D_{1}$. In a previous study, we have shown that SDS/DOH foams remain in the immobile-surface regime for viscosity $\mu$ up to ten times the viscosity of water $\mu_{\text {water }}$ [22]. Here, we show that with $\mu>16 \times \mu_{\text {water }}$, a change of regime is observed: in figure 8 , far enough from the top, we find $\beta \sim-1.7$, consistent with the mobile-surface regime.

\subsection{Bubble size $D_{2}=180 \mu \mathrm{m}$}

4.3.1. Surface rheology. When we turned to the bubbles of size $D_{2}=180 \mu \mathrm{m}$, we found results surprisingly consistent with the mobile-surface regime, for SDS and even for the SDS/DOH solution (figure 7(b)). Scaling behaviours are found with exponents increasing 


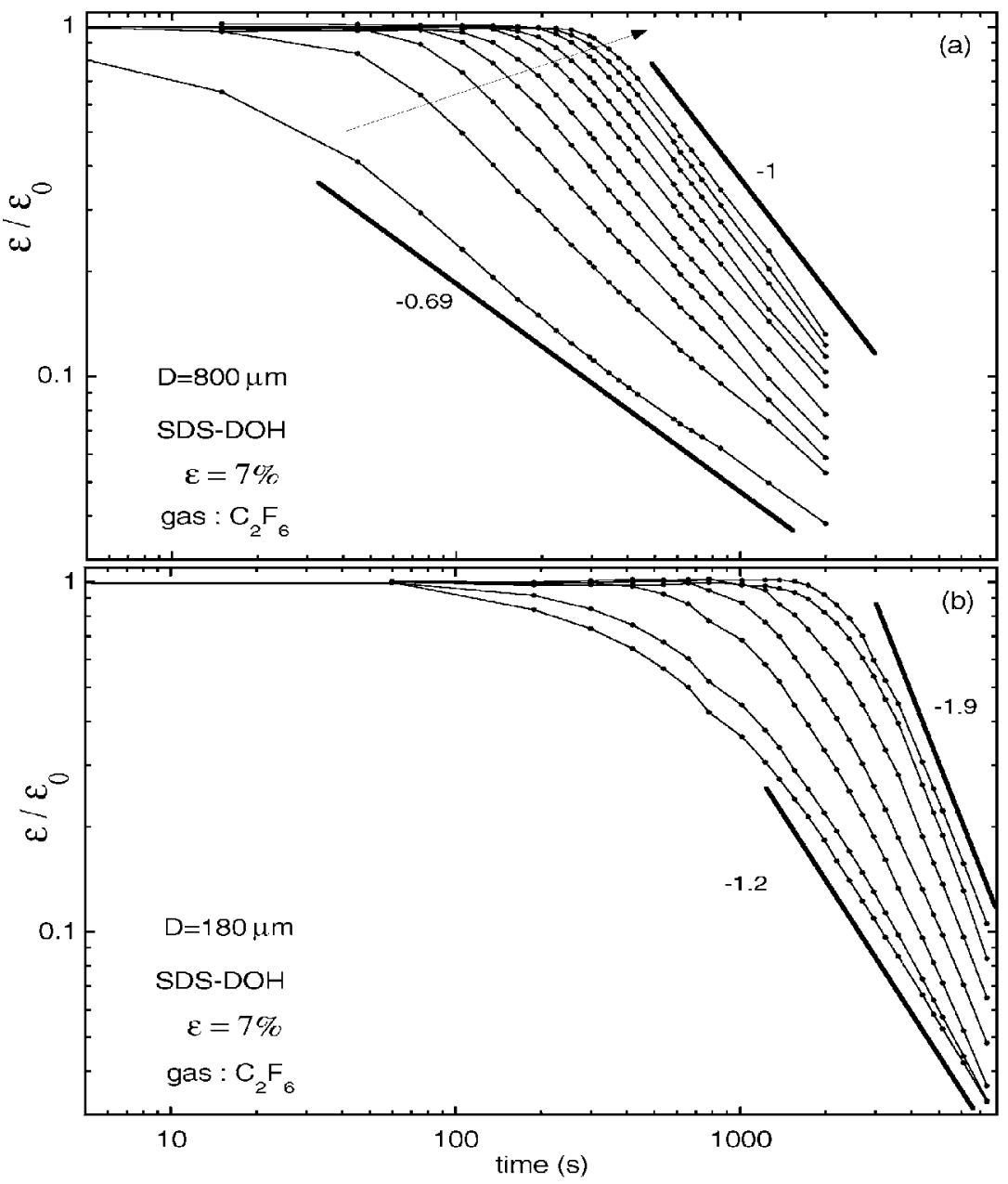

Figure 7. Normalized liquid fraction versus time, at different heights in the foam. The arrow indicates the direction from the top $(z=0)$ to the bottom. The first measurement is typically for $z=1-2 \mathrm{~cm}$, and the distance between each measurement is $\Delta z=4 \mathrm{~cm}$.

with $z$ from -1.25 to -2 . An exponent $\sim-2$ is found after a few centimetres; here again, it is consistent with the variation of $\chi$. Also, the scaling behaviour starts below $\varepsilon / \varepsilon_{0} \sim 1 / 3$, in agreement with the mobile-surface regime. Note that our interpretation arises from observations all along the foam height: measurement at a single $z$ could lead to misleading conclusions, in view of the exponent $z$-dependence (equations (4) and (6)). Indeed, exponents $\beta=-1$ can be found for mobile surfaces at small $z$, and for rigid surfaces at larger $z$. As a final test to show that coarsening is not acting in these experiments, we also performed forced-drainage experiments with bubbles of sizes $D_{1}$ and $D_{2}$ (figure 9). For an SDS/DOH foam, we find $\alpha=0.36 \pm 0.01$ with the small bubbles, whereas $\alpha=0.50 \pm 0.01$ for the large bubbles. Here, we are sure that there is almost no coarsening since the experimental time is short (the front is followed for roughly $4 \mathrm{~min}$ ) and a new foam is made before each different measurement. These results fully confirm the free-drainage observations, and show that there is a real effect of the bubble size. One can also see (figure 8) that the bubble size effect is similar to the previously described viscosity effect. 


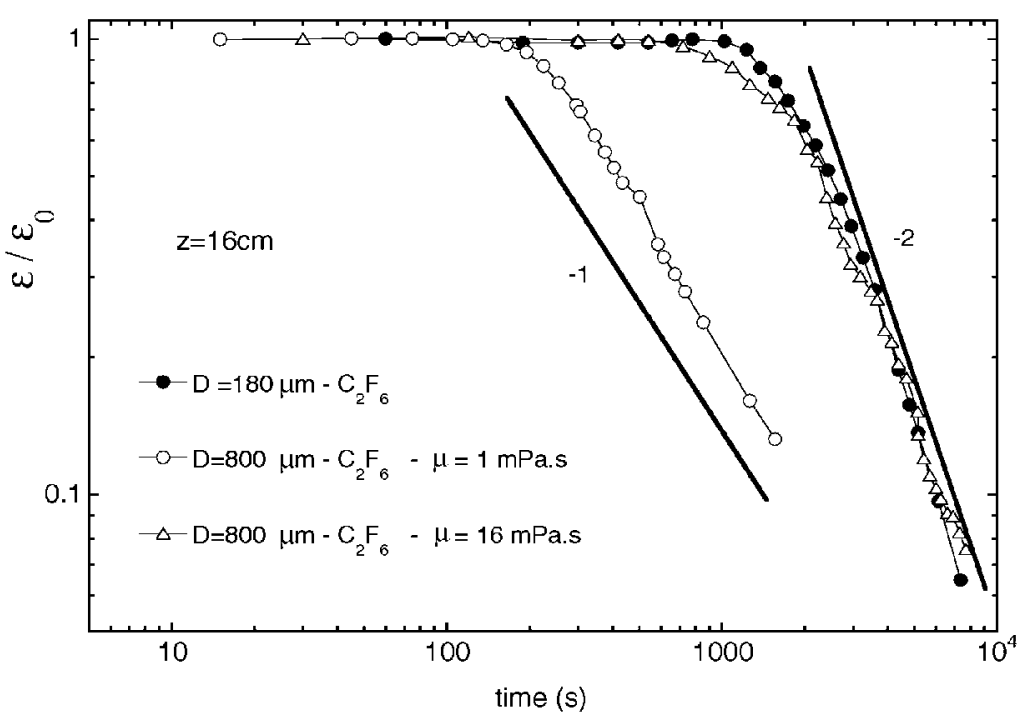

Figure 8. Normalized liquid fraction versus time, for different bulk viscosities and bubble sizes. Starting from large bubbles, an increase of the viscosity or a decrease of the bubble size provides the same effect, and the same change of power law.

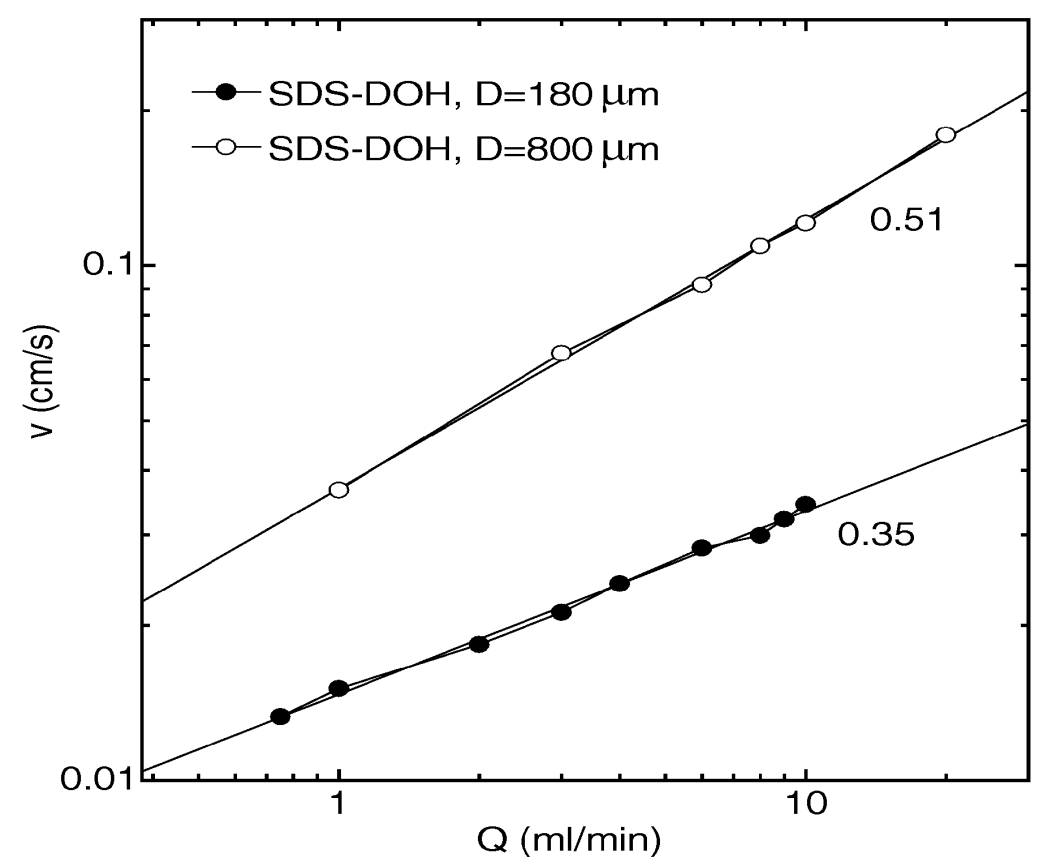

Figure 9. Forced-drainage experiments: evidence of the bubble size effect on the drainage regime.

Note that our findings are also consistent with the recent drainage study of coarsening foams of small bubbles [23], and that we can deduce from our experiments a similar value of $K_{1 / 2} \sim 0.009$. As noticed in [23], this value, which should be a constant, is surprisingly 3.5 times larger than found in [14] for bubbles of a few millimetres size. 


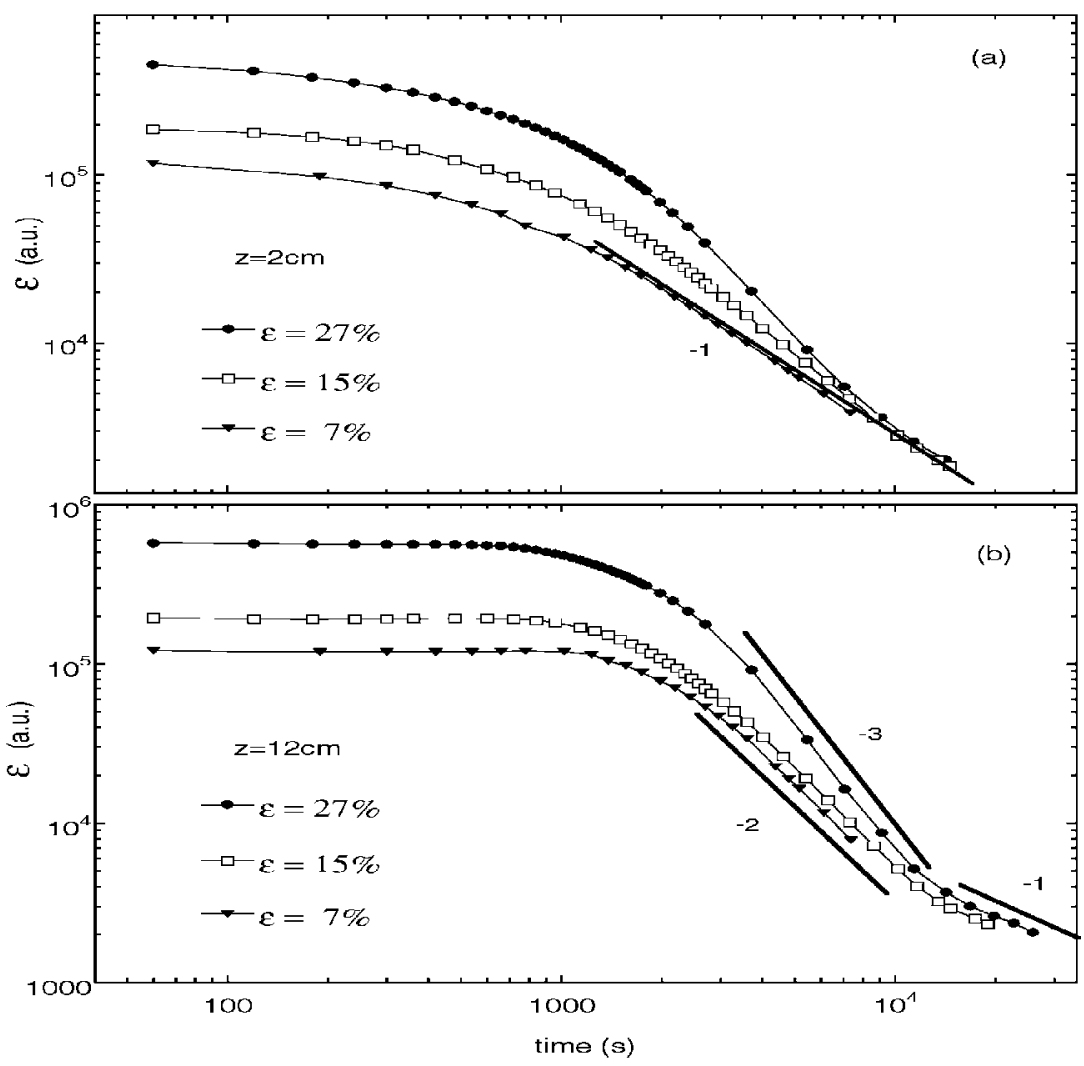

Figure 10. Liquid fraction (arbitrary units) versus time, for different initial foam wetnesses. $D(t=0)=180 \mu \mathrm{m}$

We can also add that preliminary results with protein solutions (providing extremely rigid surfaces) show that the rigid surface regime can be recovered for these small bubbles (in spite of a similar amount of coarsening). It is finally interesting to remark that once the experimental conditions are well controlled (uniform foam, no coarsening), the two experimental modesforced and free drainage_-provide the same results. Free drainage appears to be even simpler since all the measurements can be made during the drainage of a single foam, whereas forced drainage implies many different front propagations to obtain the same information.

4.3.2. High wetness. We have also investigated the limit of wet foams. All the results presented before were for foams with $\varepsilon_{0}$ between 0.04 and 0.10 . For very-high-liquid-fraction foams $(\varepsilon>0.15-0.20)$, forced drainage cannot be studied because of front instabilities $[6,35]$. Thanks to our foam production apparatus, we can create and study free drainage of such wet foams (figure 10). Close to the foam top, a power law with $\beta \sim-2$ is seen, evolving towards -1 as the liquid fraction becomes smaller. Away from the top, $\beta$ can reach -3 for the wettest foams. Nevertheless, at very long times, as $\chi$ becomes smaller than unity, an exponent -1 is recovered as expected.

The behaviour of these high- $\varepsilon$ foams does not fit with any predictions. This may be due to the inadequacy of the models, based on the existence of well defined PBs: the repartition of liquid between the PBs and the nodes changes as $\varepsilon$ increases, as well as their relative size. Moreover, coarsening effects could also have to be taken into account in these data. 
For the previous drier foams, coarsening (small but still present) is mainly active at short times, because the foam is initially already dry enough to coarsen. So, within the power law regime, the bubbles are almost no longer growing. In contrast, for the wettest foams, almost no coarsening occurs as long as $\varepsilon$ is large, and the foams mainly coarsen at longer foam ages than the dry foams, principally during the time where one sees the high-exponent power laws. However, as stated before, a small coarsening, as seen here, cannot change the drainage rates so much. Thus, the drainage behaviour of the very wet foams remains puzzling, and still has to be understood.

\section{Discussion}

Putting these new results together with previous ones allows us to test the different mobility parameters. Koehler et al [14, 27] have found direct evidence of plug flow regimes for SDS solutions, and Poiseuille flow regimes for protein solutions, with bubble sizes of a few millimetres. Preliminary results in our group with such large bubbles provide the same results: a plug flow drainage type is easily found, even with dodecanol added to SDS. From the flow profile in a PB, they have extracted values of $M_{l}$ in both cases: $M_{l} \sim 2$ for SDS solutions (corresponding to a SDS surface viscosity of $\mu_{s}=3.6 \times 10^{-5} \mathrm{~g} \mathrm{~s}^{-1}$ ), and $M_{l}<1$ for protein solutions $\left(\mu_{s}=2 \times 10^{-2} \mathrm{~g} \mathrm{~s}^{-1}\right) . M_{l}$ therefore appears to be a good parameter for describing the mobility of the interface, at these bubble sizes. Following this picture, with the same surface viscosity, it turns out that $M_{l}$ is slightly smaller than unity for our $800 \mu \mathrm{m}$ SDS bubbles. This can explain why we never reach a pure mobile surface regime for this bubble size.

In this interpretation, in order to explain the observed power laws for mobile surfaces $\left(M_{l}>1\right)$, one must consider that the mobile-surface regime is associated with the mechanism of dissipation in the nodes, since the dissipation in the surface alone probably cannot explain these scalings. We therefore come up with the following picture for the large bubble sizes: the balance between surface shear and bulk dissipation controls the boundary conditions at the surface and the surface mobility; but once the surfaces are mobile, the nodes finally control the macroscopic drainage regime.

The bulk viscosity effect that we have observed can also be explained within the same argument: since it is necessary to increase the bulk viscosity by roughly a factor of 20 to reach the fluid regime for the SDS/DOH foams, a similar ratio should exist for the surface viscosities between SDS and SDS/DOH, which seems reasonable [31].

However, the $M_{l}$ argument cannot explain our observations for the very small bubbles. For the $D_{2}$ bubbles, $M_{l}$ is always $\ll 1$. Nevertheless, the other surface effect, related to $M_{d} \sim 1 / r$, allows the flow in PBs to become pluglike for small bubbles. Unfortunately, the numerical value of $M_{d}$ is too small to account for the transition. Some hypotheses, simplifications or numerical estimations made in [21] (lubrication limit, linearized equations) are probably inadequate. The implementation of this model is an ongoing work. Nevertheless, in such an explanation for the small-bubble behaviour, dissipation in the nodes is not needed, and the specific longitudinal surface dissipation alone can explain the observed power laws. Again, it is important to remark that the bulk viscosity effect could also be explained with the $M_{d}$ argument.

Since the mobile-surface regimes seen for the very small and the very large bubbles do not have the same origin, there are no reasons why $K_{1 / 2}$ should be the same in these two cases $\left(K_{1 / 2}\right.$ is in fact a measure of the drainage velocity normalized by the bubble size, in the mobile-surface regime). This is in agreement with our measurement of $K_{1 / 2}$, which is different for the small bubbles compared with those of millimetre sizes. Moreover, the small $K_{1 / 2}$ value $(\sim 0.003)$ found for the large bubbles agrees well with viscous dissipation being larger in the nodes than in the PBs, as $K_{1 / 2}<K_{1} \sim 0.007$ [14]. This is no longer valid for the high $K_{1 / 2}$ value found 
for the small bubbles, and this shows that the node contribution is definitively too small in this regime, and that all effects originate from the PBs. In order to better understand these quantitative issues on $K_{1 / 2}$ and to fully validate the above explanations, we are now investigating in detail the bubble size dependence of the drainage velocity. Preliminary results shows that $K_{1 / 2}$ continuously decreases with increasing bubble size, but the curve shows different slopes for small and large bubbles and a kink around $D \sim 1 \mathrm{~mm}$, precisely where one can expect the crossover between the different mechanisms associated with the mobile-surface regime.

Previous and new results can thus be interpreted (at least qualitatively) and compiled in the framework of two drainage type transitions, with two parameters $M_{l}$ and $M_{d}$, related to different surface dissipation effects, and acting at different length scales. Different experimentally relevant situations can be described. For a moderate surface viscosity (SDS/DOH solution), one can have a change from plug flow to Poiseuille flow regimes, and back again to plug flow, as one decreases the bubble size. For very small or very large bubbles, surfaces have to be extremely rigid to provide Poiseuille flow within the PBs. Pure SDS solutions would almost always give a plug flow regime, but with a range of intermediate sizes where it is less marked. Note that these changes of regimes are also driven by the bulk viscosity.

\section{Conclusion}

The new results presented here show that foam drainage is complex, and depends on many parameters, related to the constituents (gas, liquid and surfactant) and to the foam sample (bubble size, wetness and height). Our investigation shows how these parameters act both on how fast the liquid drains, and-less obviously_on how it drains, with the existence of two types of drainage. We have shown that there are transitions between these regimes, in relation with the boundary conditions at the surface of the PBs. It turns out that changing (1) surface rheology, (2) bulk viscosity, (3) bubble size or (4) liquid fraction can induce changes, not simply in the drainage speed, but also in the PBs' type of flow, and thus in the macroscopic drainage type. All these effects can be compiled in terms of two complementary dimensionless numbers $M_{l}$ and $M_{d}$, describing the coupling between bulk and surface flows, setting the mobility of the PB surfaces and acting at different length scales. Using these two numbers, one can predict the type of drainage occurring in the foam, and reconcile many previous results. We also proposed a complete picture including the effect of the nodes, which appears to be coupled with the shear dissipation in the PB surfaces, and only important for the large bubbles (in agreement with quantitative results).

Beside these results, our setup combining for the first time electrical conductimetry and a multiple light scattering technique has allowed us to evidence the coupling between drainage and coarsening, and to show how strong this coupling can be in terms of drainage times, rather than in drainage rates. We have also shown that the free drainage approach can be as fruitful as forced drainage, as soon as coarsening is ruled out, and when the evolution of the liquid content is followed all along the foam sample. Within this free-drainage approach, we have also been able to study the case of very wet foams (impossible in forced-drainage experiments) and shown that new unexplained behaviours are found.

However, even if we understand now much better all these foam instabilities, there are still questions and problems: about the actual value of $M_{d}$, about the drainage of wet foams (in relation with the effective role of the nodes), about a detailed description of coarsening and of its coupling with drainage, about the balance between surface dissipation and node dissipation when $M_{l}>1$ and also about the other mechanisms of liquid transport (such as the convective instabilities). Further work is thus needed to obtain a complete picture of foam drainage and foam stability. 


\section{References}

[1] Weaire D and Hutzler S 1999 The Physics of Foams (New York: Oxford University Press)

[2] Weaire D, Pittet N, Hutzler S and Pardal D 1993 Phys. Rev. Lett. 712670

[3] Phelan R, Weaire D, Peters E A J F and Verbist G 1996 J. Phys.: Condens. Matter 8 L475

[4] Verbist G, Weaire D and Kraynik A 1996 J. Phys.: Condens. Matter 83715

[5] Weaire D, Hutzler S, Verbist G and Peters E A J F 1997 Adv. Chem. Phys. 102315

[6] Hutzler S, Weaire D and Crawford R 1998 Europhys. Lett. 41 (4) 461

[7] Hutzler S and Weaire D 2000 Phil. Mag. Lett. 80 (6) 419

[8] Cox S J, Weaire D, Hutzler S, Murphy J, Phelan R and Verbist G 2000 Proc. R. Soc. A 4562441

[9] Cox S J, Bradley G, Hutzler S and Weaire D 2001 J. Phys.: Condens. Matter 134863

[10] Cox S J and Weaire D 2001 Europhys. Lett. 55445

[11] Cox S J, Bradley G and Weaire D 2001 Eur. Phys. J. AP 1487

[12] Stoyanov S, Dushkin C, Langevin D, Weaire D and Werbist G 1998 Langmuir 144663

[13] Koehler S A, Hilgenfeld S and Stone H A 1999 Phys. Rev. Lett. 824232

[14] Koehler S A, Hilgenfeld S and Stone H A 2000 Langmuir 166327

[15] Hilgenfeld S, Koehler S A and Stone H A 2000 Phys. Rev. Lett. 864704

[16] Koehler S A, Hilgenfeld S and Stone H A 2000 Europhys. Lett. 54 (3) 335

[17] Saint-Jalmes A, Vera M U and Durian D J 1999 Eur. Phys. J. B 1267

[18] Saint-Jalmes A, Vera M U and Durian D J 2000 Europhys. Lett. 50695

[19] Saint-Jalmes A and Durian D J 2001 Europhys. Lett. 55447

[20] Durand M, Martinoty G and Langevin D 2000 Phys. Rev. E 60 R6307

[21] Durand M and Langevin D 2002 Eur. Phys. J. E 735

[22] Safouane M, Durand M, Saint-Jalmes A, Langevin D and Bergeron V 2001 J. Physique IV 11 Pr6-275

[23] Vera M U and Durian D J 2002 Phys. Rev. Lett. 88 088304-1

[24] Grassia P, Cilliers J J, Neethling S J and Ventura-Medina E 2001 Eur. Phys. J. E 6325

[25] Neethling S J, Lee H T and Cilliers J J 2002 J. Phys.: Condens. Matter 143715

[26] Gol'dfarb I I, Kann K B and Shreiber I R 1988 Fluid Dyn. 23244

[27] Koehler S A, Hilgenfeldt S, Weeks E R and Stone H A 2002 Phys. Rev. Lett. submitted

[28] Leonard R A and Lemlich R 1965 AIChE J. 1118

[29] Kraynik A 1983 Sandia Report (SAND 83-0844)

[30] Desai D and Kumar R 1982 Chem. Eng. Sci. 371361

[31] Djabbarah N F and Wasan D T 1982 Chem. Eng. Sci. 37175

[32] Lemieux P A, Vera M and Durian D J 1998 Phys. Rev. E 574498

[33] Vera M U, Saint-Jalmes A and Durian D J 2001 Appl. Opt. 404210

[34] Durian D J, Weitz D A and Pine D 1991 Phys. Rev. A 44 R7902

[35] Vera M U, Saint-Jalmes A and Durian D J 2001 Phys. Rev. Lett. 84 (13) 3001 